\title{
Perspective
}

Teresa Semalulu, Jeanine McColl, Arzoo Alam, Steffy Thomas, Julie Herrington, Jan Willem Gorter, Tania Cellucci, Stephanie Garner, Liane Heale, Mark Matsos, Karen A. Beattie and Michelle Batthish*

\section{The transition from pediatric to adult rheumatology care through creating positive and productive patient-provider relationships: an opportunity often forgotten}

https://doi.org/10.1515/jtm-2021-0001

Received January 4, 2021; accepted March 23, 2021;

published online April 15, 2021

\section{Abstract}

Background: The transition of patients with a chronic rheumatic disease from pediatric to adult care has been characterized by poor medical and patient-centered outcomes due to the lack of comprehensive transition programs and the paucity of evidence to guide practitioners. We describe a multidisciplinary transition program, data assessing patients' preparedness for transition and perception of care providers, and the association between these outcomes.

Content: Patients aged 14-19 with childhood-onset systemic lupus erythematosus (cSLE) or juvenile idiopathic arthritis (JIA) were recruited from Rheumatology Transition Clinics and Young Adult Clinics at a single institution.

*Corresponding author: Michelle Batthish, MSc, MD, FRCPC, Pediatric Rheumatologist, Associate Professor, Department of Pediatrics, McMaster University, 1280 Main St West, Hamilton, L8S 4K1, ON, Canada, Phone: +1 905521 2100, E-mail: batthim@mcmaster.ca. https://orcid.org/0000-0001-6412-1029

Teresa Semalulu, Stephanie Garner, Mark Matsos and Karen A. Beattie, Division of Rheumatology, Department of Medicine, McMaster University, Hamilton, Canada

Jeanine McColl, Department of Pediatrics, McMaster University, Hamilton, Canada

Arzoo Alam and Steffy Thomas, McMaster University, Hamilton, Canada

Julie Herrington, Tania Cellucci and Liane Heale, Division of Rheumatology, Department of Pediatrics, McMaster University, Hamilton, Canada

Jan Willem Gorter, Department of Pediatrics and Director of CanChild Centre for Childhood Disability Research, Division of Physical Medicine and Rehabilitation, McMaster University, Hamilton, Canada
Participants completed the TRANSITION-Q, which assesses healthcare self-management skills as a proxy for transition readiness, and the Consultation and Relational Empathy Scale (CARE) questionnaire, which measures patients' perception of their providers' care and empathy.

Summary: Among 63 participants, $87 \%$ had JIA (mean age 16.5 years). Age was the only patient characteristic positively associated with TRANSITION-Q scores. CARE scores revealed overwhelmingly positive interactions between patients and healthcare team members. TRANSITION-Q and CARE scores were positively correlated.

Outlook: The transition from pediatric to adult rheumatology care should be recognized as an opportunity to impact the trajectories of patients entering adult care where the patient-provider relationship may play an important role.

Keywords: patient-provider relationships; rheumatology; transition.

\section{Background}

The transition of youth and young adults with chronic disease from pediatric to adult care is often a vulnerable time in the course of a person's disease. This susceptible period has been characterized by potentially devastating medical and patient-centered outcomes due to incompatibility or inadequacy of the transition process relative to patients' needs [1, 2]. Consequently, this period must be viewed as more than a physical exchange and administrative transfer of care, but as "the purposeful, planned movement of adolescents and young adults with chronic physical and medical conditions from child-centred to adult-oriented health care systems" [3]. Youth with rheumatic disease are at-risk during this process and poor 
transition has been associated with loss to follow-up, worsening disease activity, morbidity, mortality, and other adverse outcomes. This is particularly concerning since the majority of these patients will have persistently active disease well into adulthood [4].

Davis et al. summarized findings from the literature evaluating transition care programs for pediatric patients with diabetes, sickle cell anemia, solid organ transplant, youth with long-term disability, rheumatic disease, and others [5]. In rheumatology, there has been a steady increase in the number of transition programs reported in the literature with great variability in program structure, delivery, targeted clinical population, and personnel involved [6-8]. A Cochrane Review identified four randomized control trials of transition programs for adolescents with complex medical diseases, including type 1 diabetes, spina bifida, and heart disease. Interventions included a two-day workshop, a one-on-one nurse-led teaching session, an internet-based educational intervention and a comprehensive coordinator-led program. While there may have been some improvement in patient knowledge and self-efficacy, the true impact of these programs is difficult to ascertain due to low level of evidence [9] with the majority of studies being descriptive in nature. Among these interventions, all involved a person or team in their delivery. It is unclear how much of the impact of these programs was based on the intervention itself vs. the combined impact of the patient-provider relationship and the intervention, or other factors. A qualitative study of youth with chronic health conditions that examined the different experiences patients, parents and healthcare providers on self-management revealed that youth depend on providers for support with self-management and, thus, play a critical role in promoting self-management and empowering youth to increasingly take on more responsibility for their own care [10].

Given the variability in programs and program delivery that has been reported in the literature, a standardized approach to transition care would ensure that a minimal standard of care is provided to all patients during this shift in providers. Children's Healthcare Canada (formerly known as The Canadian Association of Pediatric Health Centres [CAPHC]) established a national multidisciplinary group of healthcare providers to investigate and develop resources to address the issues faced by youth transitioning to adult care [11]. Guidelines for transition were multidimensional, focusing on patient-centered, clinical and systems level changes. Many of the same sentiments were reflected by the Agency for Healthcare
Research and Quality report through their Got Transition framework [5].

In 2016, The European League Against Rheumatism (EULAR) and Paediatric Rheumatology European Society (PReS) developed consensus-based guidelines for transitional care in youth with juvenile-onset rheumatic disease [4]. Twelve recommendations were established, including: the need to ensure high-quality coordinated multidisciplinary care delivered in partnership with the patient and their family (recommendation 1); transition should start as early as possible (recommendation 2) with a focus on developmentally appropriate care (recommendation 7); and there should be direct communication between the pediatric and adult rheumatology teams (recommendation 3). Effective provider communication and favorable patient-provider relationships have been associated with increased adherence, improved selfmanagement skills, health care satisfaction, and favorable outcomes following transition [2, 12-14]. Thus, the role of communication in transition preparedness, particularly communication between the patient and provider, is a vital component of the transition process which has potential to make significant positive impacts on the experiences and skills of patients.

Given this, we will present our comprehensive multidisciplinary rheumatology transition program and highlight the evaluation of transition readiness of youth in the context of evaluating the patient's perception of the patient-provider relationship.

\section{Content}

\section{Rheumatology Transition Clinic and the Young Adult Clinic}

Youth with childhood-onset Systemic Lupus Erythematosus (cSLE) and Juvenile Idiopathic Arthritis (JIA) are seen in our multidisciplinary Rheumatology Transition Clinic as early as the age of 14 . These patients are seen by both a pediatric and adult rheumatologist at the same time, alongside other members of the multidisciplinary care team comprised of a nurse, child life specialist and two physiotherapists, one with advanced rheumatology training (Table 1). Youth are transitioned to the Young Adult Clinic at age 18 where they are followed by the same adult rheumatologist until they are 22-24 years of age at the discretion of the treating physician. 
Table 1: Multidisciplinary team members.

\begin{tabular}{|c|c|}
\hline Team member & Role \\
\hline Adult rheumatologist & $\begin{array}{l}\text { Provides patient care concurrently } \\
\text { with the pediatric rheumatologist } \\
\text { during Transition Clinics, then inde- } \\
\text { pendently to patients age } 18-22 \\
\text { during the Young Adult Clinics. }\end{array}$ \\
\hline Pediatric rheumatologist & $\begin{array}{l}\text { Provides patient care concurrently } \\
\text { with the adult rheumatologist during } \\
\text { Transition Clinics. }\end{array}$ \\
\hline Nurse & $\begin{array}{l}\text { Provide nursing care to pediatric } \\
\text { patients. }\end{array}$ \\
\hline Child life specialist & $\begin{array}{l}\text { Pediatric specialist that works with } \\
\text { the patient and family to achieve the } \\
\text { patient's psychosocial well-being. }\end{array}$ \\
\hline Physiotherapist & $\begin{array}{l}\text { Helps patient manage sequelae of } \\
\text { their musculoskeletal disease, } \\
\text { including ways to maintain their } \\
\text { mobility. }\end{array}$ \\
\hline $\begin{array}{l}\text { Advanced Clinician Practi- } \\
\text { tioner in Arthritis Care (ACPAC) }\end{array}$ & $\begin{array}{l}\text { Physiotherapists with advanced } \\
\text { rheumatology training. }\end{array}$ \\
\hline
\end{tabular}

ACPAC, Advanced Clinician Practitioner in Arthritis Care.

\section{Participants}

Youth between the ages of 14 and 20 with cSLE or JIA were recruited from the aforementioned transition and young adult rheumatology clinics from January 2019 to March 2020. Youth who could not communicate in English, provide or lead their own care, or who were completely dependent on their caregivers for daily functioning were excluded.

\section{Data collection}

Age, sex, age at diagnosis and disease duration were obtained from patients' medical records. Participants were asked to complete two questionnaires at the time of consent:

Questionnaire 1: The TRANSITION-Q is a 14-item questionnaire used to assess non-disease specific measures of self-management in healthcare and has been validated in adolescents with chronic health conditions [15]. The questionnaire includes questions related to whether the adolescent asks or answers questions in clinic, is in charge of refilling prescriptions and taking their medications, arranges their own appointments and travels to appointments independently. Participants can respond "always", "sometimes", and "never" to each question, with a maximum achievable score of 100. This questionnaire is available through the free online application "MyTransition" (https://www.canchild.ca/ en/research-in-practice/current-studies/apply-themytransition-app-in-transition-applyit-study/ mytransition-app), which provides the participant with immediate feedback upon completion of the questionnaire by clustering each self-management skill based on their responses. The youth and a member of the clinical team identify one or two self-management skills that they have answered "sometimes" or "never" to as a goal they can work towards for their next visit.

Questionnaire 2: The Consultation and Relational Empathy (CARE) questionnaire is a validated questionnaire that was developed for primary care to measure patients' perceptions of their physician's care and empathy [16]. This 10-item questionnaire includes questions about the extent to which their provider made them feel at ease, allowed them to voice their concerns, showed compassion and involved the patient in care planning. Responses are on a five-point Likert scale (poor-excellent) with a maximum score of 50 .

\section{Analysis}

Patient characteristics and questionnaire scores were summarized using descriptive statistics. The association between each of age and disease duration and scores from the TRANSITION-Q and CARE questionnaires were determined using Spearman correlation. Multivariable linear regression determined the association between TRANSITION-Q and CARE scores after accounting for age and disease duration. Data were analyzed using Stata/IC 13.1 (2003) for Mac.

This study was approved by the Hamilton Integrated Research Ethics Board (HiREB).

\section{Results}

A total of 63 youth consented to participate in the study, among whom 55 had JIA and eight had cSLE (mean disease duration 4.6 years). The mean age (SD) was 16.5 (1.3) years (Table 2). The median TRANSITION-Q score was 61 (IQR 42-50). The median CARE score was 47 (IQR 42-50).

Median TRANSITION-Q scores appeared to increase with age. Age was the only patient characteristic that was associated with TRANSITION-Q scores (Spearman's 
Table 2: Baseline characteristics $n=63$.

\begin{tabular}{lrr}
\hline & Mean (SD) or $\mathbf{n}(\%)$ & Range \\
\hline Age, years & $16.5(1.3)$ & $14-20$ \\
Female & $41(65)$ & \\
Diagnosis & & \\
$\quad$ II & $55(87)$ & \\
JSLE & $8(13)$ & \\
Age at diagnosis, years & $11.9(4.5)$ & $1-18$ \\
Disease duration, years & $4.6(4.5)$ & $0-16$ \\
\hline & Median & IQ range \\
\hline & 47 & $42-50$ \\
\hline CARE scores ${ }^{\mathrm{a}}$ & 61 & $50-70$ \\
TRANSITION-Q scores ${ }^{\mathrm{b}}$ & 51.5 & $50-56$ \\
14 years $(\mathrm{n}=6)$ & 58 & $(47-63)$ \\
15 years $(\mathrm{n}=5)$ & 58 & $(44-68)$ \\
16 years $(\mathrm{n}=19)$ & 64 & $(53=78)$ \\
17 years $(\mathrm{n}=22)$ & 70 & $(58-70)$ \\
18 years $(\mathrm{n}=9)$ & 75 & - \\
19 years $(\mathrm{n}=1)$ & 92 & - \\
20 years $(\mathrm{n}=1)$ &
\end{tabular}

${ }^{a}$ Maximum possible CARE Score is $50 .{ }^{b}$ Maximum possible TRANSITION-Q score is 100. CARE, Consultation and Relational Empathy Scale.

rho $=0.408, p=0.001)$. There was an association between TRANSITION-Q scores and patient-perceived relationship with their providers (Spearman's rho $=0.306, p=0.015$ ), which persisted after including age and age at diagnosis into the model (standardized $\beta=0.252, p=0.032$ ) (Figure 1).

\section{Summary}

The multidisciplinary transition team in rheumatology at McMaster University highly values communication and partnership with patients and families as key components of supporting youth as they improve their self-management skills in preparation for the transfer to adult care. In recognizing and integrating the key components of guidelines for transitional care into our practice, we recognized that understanding the variation in patients' skills and strengths in self-management were necessary to guide the development of targeted tools or strategies to improve transition preparedness and, ultimately, patient outcomes [4]. We have incorporated a tool to measure readiness for transition in our transition clinic as a means of helping team members identify areas for improvement, strategize ways to support the youth, and identify gaps/barriers in care.

Our multidisciplinary transition program enrolls patients as early as age 14 or, in the case of patients newly diagnosed after age 14, soon after the time of diagnosis. While regularly followed by their pediatric rheumatologist until the age of 18 , they are seen annually (14-15 years old) or regularly as needed (16-18 years old) in the transition clinic where the pediatric and adult rheumatologists see the patient together at the same time before transitioning to the Young Adult clinic. We use the TRANSITION-Q questionnaire clinically as a proxy to assess transition preparedness and healthcare self-management skills. This allows us to identify areas for improvement and to initiate dialog and goal setting with the youth. In measuring transition readiness, we can investigate the importance of age as a primary driver for transition preparedness. In this study, we observed a wide variation in self-management skills among patients of the same age. This reinforces the need to initiate transition at an early age and to tailor programs or strategies to the developmental stage of each youth in order to optimize self-management skills.

Our study also revealed favorable perceptions of the patient-provider relationship which increased with age.

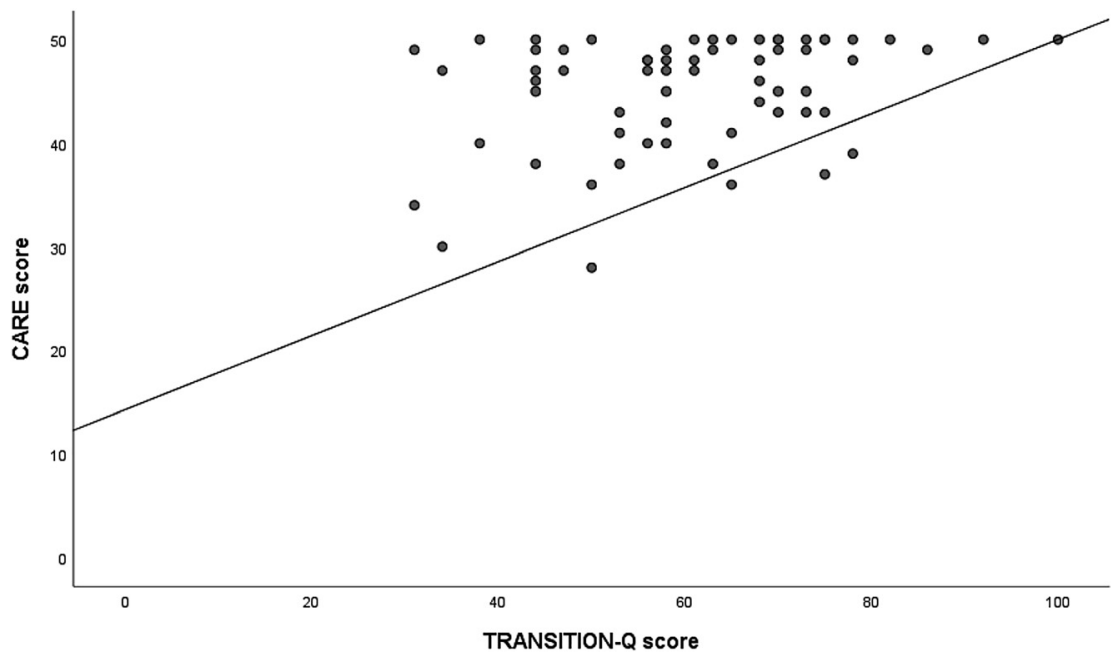

Figure 1: Association between TRANSITION-Q and CARE scores. 
We also found a positive relationship between transition readiness and CARE scores. Although the majority of patients expressed very positive relationships and interactions with their healthcare team as shown by the care scores, our data suggest that there is a positive association between the patient-provider relationship and transition readiness where a favorable relationship between patients and providers correlates with a higher level of healthcare self-management skills [17]. A trusting relationship between a youth and healthcare provider may not only impact patients' desire and ability to actively participate in their care, but also the ability of the clinician to provide individualized care to their patients, both which will increase the likelihood of a successful transition $[17,18]$. Thus, the patient-provider relationship should not be overlooked as a key component of transition preparedness.

The core elements of the Got Transition framework, as they relate to health care transition, include transition policy, transition preparation, transition planning, transition and transfer of care, and transition completion [5]. Many of these elements overlap with the EULAR/PReS guidelines [4]. The triple aim approach is commonly cited as framework for evaluating these processes in terms of [1] the patient experience of care [2], population health outcomes and [3] per capita cost [19]. There is universally a great deal of work needed to implement much needed change, as coordinated efforts to promote change at the healthcare system level are required to truly bridge the gap between pediatric and adult care. Transitional care programs protect young patients by equipping them with the skills necessary to navigate the system despite its flaws. Additionally, focusing on the aim of optimizing the patient experience requires attention to be paid to the delivery of transition programs and the quality of the patient-provider relationship. The main limitation of this study is the modest sample size, which limits our ability to make robust conclusions from our data. However, data collection is ongoing and these initial findings are promising.

\section{Reflections on the transition to adult rheumatology in a multidisciplinary rheumatology clinic}

The promising findings illustrated in this study may be attributed to multiple factors. Our perspective on these factors follows.

\section{a. The multidisciplinary care model:}

Providing care through multidisciplinary teams allows for individual patient needs to be met from a holistic perspective with various gradients of expertise. This particular care team (Table 1) addresses several domains of health care, including medical care, physical health, and psychosocial well-being. This care model also builds in a redundancy of interactions with multiple health care providers, which fosters a greater sense of support for the patient and contributes to a positive patient-provider relationship. Furthermore, repeat messaging from multiple care team members reinforces important concepts, such as self-management skills needed for a successful transition to adult rheumatology. These aspects of the multidisciplinary care team likely contributed to the positive associations between the patient-provider relationship and transition preparedness seen in our data.

b. The presence of both the pediatric and adult rheumatologist in the transition clinic:

Both the pediatric and adult rheumatologist are present in the Rheumatology Transition Clinic, where patients 14-18 years are seen. This is beneficial for several reasons. A cohesive front between rheumatologists likely extends the trust that the patient has for their pediatric rheumatologist to their future adult rheumatologist. This allows the patients to build a relationship with the adult rheumatologist while maintaining their potentially long-standing relationship with their pediatric rheumatologist. Next, having the adult rheumatologist involved in the care prior to transfer allows for a greater understanding of the trajectory of the patient's disease, resulting in a smoother medical transfer of care and perhaps less abrupt changes to management at the time of transfer. This seamless transition to adult rheumatology most likely enhances patients' perception of their provider and care received, and perhaps reduces the chance that patients will be lost to follow-up. We will follow this cohort of patients over time to see how the patientprovider relationship changes at the point of transition, among other follow-up periods.

c. Goal setting performed with multidisciplinary team member:

The TRANSITION-Q questionnaire is used for goal setting, but this task is not left for the patient to figure out on their own. Goal setting is done alongside a Physiotherapist or Child Life Specialist, who encourages the patient to identify areas to work on and provides guidance as to how they can work towards developing skills in their self-identified areas of 
improvement. This process allows patients to develop necessary self-management skills with a sense of independence, while feeling supported. To date, we have already observed an increase in TRANSITION-Q scores overtime with goal setting among a small subset of our cohort with multiple follow-up visits.

\section{d. Patients enter transition clinic at an early age:}

Patients can start the transition process as early as age 14. This is in keeping with EULAR/PRES recommendations, which state that the "transition process should start as early as possible; in early adolescence or directly after the diagnosis in adolescent-onset disease" [7]. An early transition provides ample time for patients to prepare for their transfer to adult rheumatology. This process not only allows for the patient to mature and develop self-management skills but gives patients time to become accustomed to the upcoming change in their care, which may help to prevent poor outcomes during this vulnerable period.

After age 16, patients are seen in the transition clinic every three-six months at the discretion of the physician. Once they turn age 18, they are transferred to adult care where they are followed in the Young Adult Clinic with the same frequency. This is not routinely done in adults care settings. The frequency by which patients are followed provides consistency throughout the transition process and not only allows close monitoring of disease activity but provides multiple opportunities to work on selfmanagement skill development, builds trust between the patient and their provider, and maintains a routine to prevent lost to follow-up as they transfer care.

\section{Outlook}

The patient-provider relationship should be recognized as an opportunity to positively influence the disease course of young patients entering the adult healthcare system. Our results imply that interventions to improve transition readiness should focus on enhancing self-management skills in a way that patients feel cared for and trusted. As patients mature, they will naturally become more prepared for the transition process; however, the unfamiliarity with adult healthcare system can have negative consequences on this growth. Coordinated efforts by a multidisciplinary team that includes both pediatric and adult rheumatologists, can help patients appropriately apply the skills they have acquired to navigate the adult health care system with the independence required to be successful.
As we move towards prioritizing transition programs in rheumatic disease, we need to generate high-quality evidence to ensure a minimum standard of care is provided to all patients. Future steps will need to identify meaningful outcomes to define transition success, as there still exists variation in how successful transitions are defined.

Acknowledgments: We would also like to thank Debbie Poppa, Jennifer Zuccolo (Physiotherapist), Heather McKean (Child Life Specialist) for their contributions towards the study. Dr. Gorter holds the Scotiabank Chair in Child Health Research.

Research funding: Michelle Batthish was supported by a New Investigator Fund at Hamilton Health Sciences. This research is also funded by the Arthritis Society's Arthritis Centre Grant (AC-19-0517).

Author contributions: All authors have accepted responsibility for the entire contents of this manuscript and approved its submission.

Competing interests: Authors state no conflict of interest. Informed consent: Informed consent was obtained from all individuals included in this study.

Ethical approval: Research involving human subjects complied with all the relevant national regulations, institutional policies and is in accordance with the tenets of the Helsinki Declaration (as revised in 2013) and has been approved by the authors' institutional review board (\#5479).

\section{References}

1. Felsenstein S, Reiff AO, Ramanathan A. Transition of care and health-related outcomes in pediatric-onset systemic lupus erythematosus. Arthritis Care Res 2015;67:1521-8.

2. Wicklow BA, Sellers EA. Medical systems, patient-provider relationships, and transition of care. In: Delamater A, Marrero D, editors. Behavioral diabetes. Cham: Springer; 2020:199-212 pp.

3. Blum RW, Garell D, Hodgman CH, Jorissen TW, Okinow NA, Orr DP, et al. Transition from child-centered to adult health-care systems for adolescents with chronic conditions. A position paper of the Society for Adolescent Medicine. J Adolesc Health 1993;14:570-6.

4. Foster HE, Minden K, Clemente D, Leon L, McDonagh JE, Kamphuis $S$, et al. EULAR/PReS standards and recommendations for the transitional care of young people with juvenile-onset rheumatic diseases. Ann Rheum Dis 2017;76:639-46.

5. Davis AM, Brown RF, Taylor JL, Epstein RA, McPheeters ML. Transition care for children with special health care needs. Pediatrics 2014;134:900-8.

6. McDonagh JE, Farre A. Are we there yet? An update on transitional care in rheumatology. Arthritis Res Ther 2018;20:5.

7. McDonagh JE, Farre A. Transitional care in rheumatology: a review of the literature from the past 5 years. Curr Rheumatol Rep 2019; 21:57. 
8. Clemente D, Leon L, Foster H, Minden K, Carmona L. Systematic review and critical appraisal of transitional care programmes in rheumatology. Semin Arthritis Rheum 2016; 46:372-9.

9. Campbell F, Biggs K, Aldiss SK, O'Neill PM, Clowes M, McDonagh J, et al. Transition of care for adolescents from paediatric services to adult health services. Cochrane Database Syst Rev 2016;4: CD009794.

10. Nguyen T, Henderson D, Stewart D, Hlyva O, Punthakee Z, Gorter JW. You never transition alone! Exploring the experiences of youth with chronic health conditions, parents and healthcare providers on self-management. Child Care Health Dev 2016;42: 464-72.

11. Canadian Association of Pediatric Health Centres (CAPHC), National Transitions Community of Practice. A guideline for transition from paediatric to adult health care for youth with special health care needs: a national approach; 2016. https:// www.childhealthbc.ca/sites/default/files/caphc_transition_to_ adult_health_care_guideline_may_2017.pdf.

12. Ritholz MD, Wolpert H, Beste M, Atakov-Castillo A, Luff D, Garvey KC. Patient-provider relationships across the transition from pediatric to adult diabetes care: a qualitative study. Diabetes Educat 2014;40:40-7.

13. Monaghan M, Hilliard M, Sweenie R, Riekert K. Transition readiness in adolescents and emerging adults with diabetes: the role of patient-provider communication. Curr Diabetes Rep 2013; 13:900-8.

14. Heisler M, Bouknight RR, Hayward RA, Smith DM, Kerr EA. The relative importance of physician communication, participatory decision making, and patient understanding in diabetes selfmanagement. J Gen Intern Med 2002;17:243-52.

15. Klassen AF, Grant C, Barr R, Brill H, Kraus de Camargo O, Ronen $\mathrm{GM}$, et al. Development and validation of a generic scale for use in transition programmes to measure self-management skills in adolescents with chronic health conditions: the TRANSITION-Q. Child Care Health Dev 2015;41:547-58.

16. Mercer SW, Maxwell M, Heaney D, Watt GC. The consultation and relational empathy (CARE) measure: development and preliminary validation and reliability of an empathy-based consultation process measure. Fam Pract 2004;21:699-705.

17. Stollon NB, Paine CW, Lucas MS, Brumley LD, Poole ES, Peyton T, et al. Transitioning adolescents and young adults with sickle cell disease from pediatric to adult health care: provider perspectives. J Pediatr Hematol Oncol 2015;37:577-83.

18. Reiss JG, Gibson RW, Walker LR. Health care transition: youth, family, and provider perspectives. Pediatrics 2005;115:112-20.

19. Stiefel M, Nolan K. A guide to measuring the triple aim: population health, experience of care, and per capita cost. IHI Innovation Series white paper. Cambridge, Massachusetts: Institute for Healthcare Improvement; 2012. (Available on www.IHI.org). 